\title{
Review
}

\section{Idiopathic pulmonary fibrosis in West Highland white terriers: An update}

\author{
Cécile Clercx*, Aline Fastrès, Elodie Roels \\ Department of Clinical Sciences, FARAH, Faculty of Veterinary Medicine, University of Liège, Liège, Belgium
}

\section{A R T I C L E I N F O}

Keywords:

Canine idiopathic pulmonary fibrosis

Dog

West Highland white terrier

\begin{abstract}
A B S T R A C T
Canine idiopathic pulmonary fibrosis (CIPF) affects middle-aged to older dogs of a single breed, mainly the West Highland white terrier (WHWT), which is suggestive of a genetic predisposition. CIPF causes exercise intolerance, restrictive dyspnoea and coughing. Coarse crackles are heard on thoracic auscultation. Abnormal blood gas parameters and a shortened '6-min-walking test' distance are common; secondarily induced pulmonary hypertension and/or airway collapse are frequent. These features of CIPF mimic those of idiopathic pulmonary fibrosis (IPF) in humans and therefore identify CIPF as a possible spontaneously arising model for study of human IPF. However, computed tomographic and histopathological findings of CIPF are not identical to those of human IPF. As in human IPF, the aetiology of CIPF is not yet fully elucidated. There are no curative treatments and the prognosis is poor. This paper reviews advances in understanding of the clinical description and natural history of CIPF, the investigation of biomarkers and the exploration of possible aetiologies and mechanistic hypotheses.
\end{abstract}

(c) 2018 Elsevier Ltd. All rights reserved.

\section{Introduction}

Canine idiopathic pulmonary fibrosis (CIPF) is a spontaneously arising chronic and progressive fibrotic lung disease of unknown aetiology that affects older West Highland white terriers (WHWTs). Corcoran et al. (1999a) initially described the condition in a cohort of 29 WHWTs with marked inspiratory crackles audible on auscultation of the thorax in association with interstitial changes radiographically. Additional case reports and case series were published and allowed improved clinical characterisation of this disease (Webb and Armstrong, 2002; Norris et al., 2005; Corcoran et al., 2011; Heikkilä et al., 2011). The clinical entity has been referred as chronic pulmonary disease (Corcoran et al., 1999a; Schober and Baade, 2006), chronic idiopathic pulmonary fibrosis (Lobetti et al., 2001; Webb and Armstrong, 2002), idiopathic pulmonary fibrosis (Norris et al., 2002), interstitial lung disease (Norris et al., 2005; Reinero and Cohn., 2007) and, more recently, CIPF (Johnson et al., 2005; Heikkilä et al., 2011).

This spontaneously arising fibrotic pulmonary disease shares several clinical and pathogenic features with human IPF and has been identified by some authors as a possible model for human IPF, with the hope that investigation of CIPF would offer a tool for studying some aspects of the fibrotic mechanisms that might also

\footnotetext{
* Corresponding author.

E-mail address: cclercx@uliege.be (C. Clercx).
}

prove useful for human medicine. Such perspectives, in addition to the interest in the condition in dogs, and in particular for the WHWT breed, offer an additional incentive for in-depth investigation of possible pathways leading to fibrosis in CIPF.

The aim of this narrative review is to provide an overview of published studies related to CIPF.

\section{Prevalence and clinical presentation}

The prevalence of CIPF is currently unknown.

CIPF affects mainly middle-aged to older WHWTs, with a mean age at diagnosis ranging from 9 to 13 years (Corcoran et al., 1999a; Heikkilä et al., 2011; Roels et al., 2018). Clinical signs may appear earlier in rare cases, with the earliest onset described at 2 years of age (Johnson et al., 2005; Schober and Baade., 2006). No sex predisposition has been reported (Heikkilä-Laurila and Rajamäki, 2014). Rare cases of pulmonary fibrosis have also been described in other terrier breeds, such as the American Staffordshire terrier, the Bull terrier, the Cairn terrier and the Scottish terrier (Corcoran et al., 1999b; Lobetti et al., 2001; Norris et al., 2002; Johnson et al., 2005; Krafft et al., 2013). However, only very limited data are available for these other breeds and whether pulmonary fibrosis in these dogs is the same as in the WHWT breed has not been investigated.

In WHWTs with CIPF, the disease generally develops slowly and dogs deteriorate progressively over time (Corcoran et al., 1999a). Early signs are often attributed by the owners to the normal ageing 
process (Corcoran et al., 1999a). In the majority of cases, history reveals exercise intolerance alone or in association with chronic cough in an otherwise alert and bright dog; more pronounced respiratory signs may also be reported, such as tachypnoea, excessive panting, dyspnoea, cyanosis or syncope (Heikkilä-Laurila and Rajamäki, 2014). The duration of clinical signs at the time of diagnosis also varies between individuals and ranges from a few weeks to several months (Heikkilä-Laurila and Rajamäki, 2014).

One of the most typical clinical features is inspiratory crackles on thoracic auscultation, which occurs in the majority of the affected dogs. In some dogs, crackles can even be heard without a stethoscope when the dog is breathing with an open mouth (Heikkilä-Laurila and Rajamäki, 2014). Other common clinical examination findings include positive laryngo-tracheal reflex, tachypnoea and excessive abdominal breathing (Heikkilä-Laurila and Rajamäki, 2014). The presence of wheezes on thoracic auscultation, dyspnoea and cyanosis has also been reported (Corcoran et al., 1999a).

\section{Understanding the pathogenesis}

In recent decades, research efforts have been oriented to understanding the pathogenesis of human IPF. Previously defined as an inflammatory disease, IPF is currently considered as an epithelium-driven disease, in which a dysfunctional ageing lung epithelium is exposed to recurrent micro-injuries leading to defective attempts to regenerate and aberrant epithelial-mesenchymal crosstalk (Daccord and Maher, 2016). Multiple studies have shown that alveolar epithelial cell (AEC) apoptosis secondary to injury is followed by extravascular coagulation, immune system activation and aberrant persistent activation of AECs. These cells, in turn, induce the migration and proliferation of local fibroblasts, recruit circulating fibrocytes to areas of injury, and promote differentiation of fibroblasts into persistently activated myofibroblasts, which secrete excessive amounts of extracellular matrix (ECM) proteins. This pathological cascade involves complex cellcell and cell-matrix interactions through numerous biochemical mediators, such as growth factors, enzymes, chemokines, coagulation factors and reactive oxygen species, all of which have the potential to be influenced by numerous host and environmental factors. TGF- $\beta$, a potent pro-fibrotic mediator involved in cell recruitment, myofibroblast differentiation and induction of ECM production, is cardinal among these.

In dogs, two studies have investigated how TGF- $\beta 1$ may be involved in CIPF (Krafft et al., 2014; Lilja-Maula et al., 2014b). The first aimed to investigate the expression of known extracellular matrix protein regulators of TGF- $\beta 1$ storage and activation, namely latent TGF- $\beta$ binding protein-1 (LTBP-1) and fibrillin-2, and expression of the phosphorylated signalling protein Smad2 (P-Smad2) (Lilja-Maula et al., 2014b). These authors reported that P-Smad2 immunoreactivity, an indicator of TGF- $\beta 1$ signalling activity, was increased in WHWTs with CIPF in comparison with healthy control WHWTs, suggesting enhanced TGF- $\beta 1$ signalling in CIPF as part of the pathophysiology of the disease. The second study aimed to evaluate TGF- $\beta 1$ biochemical pathways in healthy dogs and dogs with CIPF (Krafft et al., 2014), using immunohistochemistry and qRT-PCR to measure expression of TGF- $\beta 1$ and molecules involved in its storage (LTBP-1, -2 and -4 ), activation $(\alpha v \beta 6$ and $\alpha v \beta 8$ integrins, thrombospondin- 1$)$ and signal inhibition (Smad7). Results suggested that some of the activating, storage and signalling pathways of TGF- $\beta 1$ are modified in WHWTs with CIPF and may play a role in the pathogenesis of the disease.

Lilja-Maula et al. (2015) investigated the expression of activins $A$ and $B$ (cytokines belonging to the TGF- $\beta$ superfamily) in normal canine lung tissue and in lung tissue from WHWTs with CIPF, and analysed activin B in the BALF from healthy WHWTs and WHWTs with CIPF. Activin B was suggested to play a role in fibrosis and in pathological alteration of the alveolar epithelium.

\section{Aetiology and risk factors}

In people, IPF is believed to arise in genetically susceptible individuals as a consequence of an aberrant wound-healing response following repetitive alveolar injury. The exact triggers initiating the fibrotic process remain unknown. Several risk factors have been associated with disease development and progression, although their role in the pathogenesis of the disease remains unclear. These risk factors include the following: genetic background; inhalation of cigarette smoke; environmental or occupational exposure to metal, wood, sand, stone or silica dust (Raghu, 2011; Olson and Swigris, 2012); gastro-oesophageal reflux (Minnis et al., 2016); and exposure to infectious agents, including viruses (e.g. Epstein-Barr virus), bacteria or fungi (Raghu, 2011; Molyneaux and Maher, 2013; Antoniou et al., 2014).

In CIPF, in an attempt to recognise potential triggers, an online questionnaire-based survey was launched via social media to WHWT owners (Roels et al., 2018). The parameters significantly associated with CIPF included genetic relationship to a dog affected by CIPF, living in an old house, absence of a ventilation system, and frequent grooming in dedicated grooming facilities. These results indicated an association between CIPF, genetic background and environmental factors.

In CIPF, the possible role of gastroesophageal reflux and microaspiration of small amounts of gastric content was evaluated by measuring total bile acids in BALF by mass spectrometry in healthy WHWTs and WHWTs with CIPF, in comparison with BALF from dogs with other diseases (Maatta et al., 2018). Bile acids were detected more frequently in healthy WHWTs, compared with dogs of other breeds and were at higher concentrations in WHWTs with CIPF than in healthy WHWTs, suggesting that micro-aspiration could be associated with the predisposition of WHWT to CIPF.

Limited studies have investigated a possible association between CIPF and infectious agents. To assess the potential association between CIPF and canine herpesvirus infection, a PCR assay was used to test lung and blood samples from WHWTs with CIPF and control dogs without respiratory disease. Canine herpesvirus could not be amplified from the lung and blood samples from WHWTs with CIPF or samples from control dogs, making an association between CIPF and herpesvirus infection very unlikely (Roels et al., 2016).

As mentioned above, CT and histopathological findings in CIPF share characteristics of both human UIP and NSIP patterns, the latter being commonly observed in chronic hypersensitivity pneumonitis (HP), which is recognised as the main differential for IPF (Neurohr and Behr., 2015). As cases of chronic HP have been associated with a fungal aetiology in humans (Spagnolo et al., 2015; Kouranos et al., 2017), a recent study aimed to investigate a potential association between fungal infection and CIPF. Lung specimens from WHWTs with CIPF and healthy WHWTs were screened for fungal DNA using a conventional pan-fungal PCR assay targeting the conserved internal transcribed spacer (ITS) region (Roels et al., 2017b). In the same study, serum samples from the WHWTs with CIPF and from age-matched unaffected control dogs were tested for precipitins from 10 species of environmental fungi using electrosyneresis on cellulose acetate (Roels et al., 2017b). PCR results suggested that an association between CIPF and invasive fungal infection or heavy fungal colonisation was unlikely, while the higher proportion of positive precipitin reactions observed in dogs with CIPF might suggest a lung sensitisation to inhaled fungal allergens. 


\section{Genetic background}

In human IPF, several genome-wide association studies have identified several common and rare genetic variants in more than a dozen loci on different chromosomes that appear to contribute to the risk of developing IPF (Fingerlin et al., 2013; Sgalla et al., 2016; Allen et al., 2017).

In CIPF, despite of the strong predisposition of the WHWT breed to CIPF, which raises suspicion for a genetic basis (Heikkilä-Laurila and Rajamäki, 2014), and despite the clear association between CIPF and genetic background (Roels et al., 2018), genetic studies have so far failed to identify clear candidate genes.

\section{Diagnostic testing}

Accurate diagnostic testing is very challenging because of the absence of highly sensitive and specific testing modalities for CIPF. The most frequently used diagnostic tests (including haematological and serum biochemical analyses, radiography, blood gas assessment, bronchoscopy and bronchoalveolar lavage analysis, echocardiography and the '6-min walking test'; 6MWT) are not able to confirm the diagnosis, but are used to increase the index of suspicion for CIPF and to rule out comorbidities. By analogy with human medicine, high-resolution computed tomography (HRCT) and histopathology are considered to be the most specific tests to confirm the diagnosis. However, HCRT findings in CIPF fail to parallel HRCT findings in human IPF (classically characterised by a so-called 'usual interstitial pneumonia' [UIP] pattern) (Roels et al., 2017a). While the definitive diagnosis of CIPF relies ultimately on histopathology (Heikkilä-Laurila and Rajamäki, 2014), ante-mortem lung biopsy is not performed routinely in veterinary practice due to the invasiveness of the procedure. Moreover, information obtained from focal biopsy samples may not be representative of the organ as a whole, especially in view of the spatial heterogeneity of affected lungs. Again, histopathological findings in CIPF do not parallel the typical UIP histological pattern described classically in human IPF (Syrjä et al., 2013).

In human medicine, the diagnosis of IPF remains challenging and most cases need to be discussed by a multidisciplinary group. A confident diagnosis of IPF can be made in the correct clinical context when thoracic HRCT images show a pattern of typical or probable UIP (Lynch et al., 2018). If the clinical context is indeterminate for IPF, or the computed tomography (CT) pattern is not indicative of typical or probable UIP, biopsy should be considered to confirm the presence of the UIP histological pattern, and a confident diagnosis of IPF can then be made on the basis of a multidisciplinary evaluation. The same approach can be advised in canine medicine for the diagnosis of CIPF.

\section{Blood analyses}

Haematological and serum biochemical analyses are generally unremarkable in WHWTs with CIPF, but are useful in the initial diagnostic investigation to rule out other possible causes of exercise intolerance (Heikkilä-Laurila and Rajamäki, 2014).

\section{Cardiopulmonary function tests}

Diagnostic tools allowing the investigation of pulmonary function are limited in dogs in comparison with those available in human medicine, due to the impracticality of performing conscious testing. The only two tests that have been investigated for the overall assessment of the cardiopulmonary function in WHWTs with CIPF are arterial blood gas analysis and the 6MWT.

Hypoxaemia is a common finding in WHWTs with CIPF. One study reported that $45 \%$ of dogs had mild hypoxaemia (partial pressure of oxygen in arterial blood $\left.\left(\mathrm{PaO}_{2}\right), 60-80 \mathrm{mmHg}\right)$ and a further $45 \%$ had severe hypoxaemia $\left(\mathrm{PaO}_{2}<60 \mathrm{mmHg}\right.$ ) (HeikkiläLaurila and Rajamäki, 2014). In comparison with control WHWTs, WHWTs with CIPF have reduced arterial $\mathrm{PaO}_{2}$ values and increased alveolar to arterial oxygen gradient $\left(\mathrm{P}(\mathrm{A}-\mathrm{a}) \mathrm{O}_{2}\right)$, but there is no difference between groups for the arterial partial pressure of carbon dioxide $\left(\mathrm{PaCO}_{2}\right)$ (Heikkilä-Laurila and Rajamäki, 2014). However, alterations to arterial blood gases are not specific for CIPF and other causes of ventilation-perfusion mismatch or right-to-left shunting (Balakrishnan and King, 2014) should be excluded. Consequently, arterial blood gas analysis in WHWTs with CIPF is generally used to quantify the severity of the cardiorespiratory system dysfunction, rather than to confirm the diagnosis (Balakrishnan and King, 2014). Interestingly, despite such low oxygen levels, most of the WHWTs with CIPF are bright, alert, and not dyspnoeic, suggesting that slow disease progression enables the dogs to adapt to decreasing blood oxygen levels (HeikkiläLaurila and Rajamäki, 2014).

The 6MWT is a submaximal exercise test that measures the distance an individual is able to walk over 6 min (6MWD) (LiljaMaula et al., 2014a). This test is used widely in human clinical practice to assess disease progression and response to treatment, as well as in therapeutic trials to serve as a primary end-point, as it is inexpensive, convenient and well-tolerated (Demir and Küçükoglu, 2015). In WHWTs with CIPF, the 6MWT distance was significantly decreased in comparison with controls and had a moderate positive correlation with $\mathrm{PaO}_{2}$, suggesting that the test can serve as a non-invasive means of monitoring lung function and exercise tolerance (Lilja-Maula et al., 2014a).

\section{Echocardiography}

Echocardiography is an essential complementary examination in the diagnostic approach to CIPF. It allows exclusion of any concomitant primary cardiac disease and, most importantly, it quantifies pulmonary arterial pressure (PAP) to assess for secondary pulmonary hypertension $(\mathrm{PH})$, necessary for making therapeutic decisions. PH is frequent in WHWTs with CIPF (Schober and Baade, 2006; Roels et al., 2014). In the absence of cardiac catheterisation, Doppler flow interrogations of tricuspid or pulmonary insufficiency jets are the current echocardiographic reference standard for the diagnosis of $\mathrm{PH}$ in dogs. Other echocardiographic measures for a non-invasive diagnostic approach to $\mathrm{PH}$ have been developed, including acceleration time to ejection time ratio of the pulmonary artery flow (AT:ET) (Schober and Baade, 2006), the main pulmonary artery diameter to aortic diameter ratio (MPA/Ao) (Serres et al., 2007) and the right pulmonary artery distensibility index (RPAD Index) (Visser et al., 2016). Moreover, a new and promising parameter, the right pulmonary vein to pulmonary artery ratio (PV/PA), was useful in the detection of pulmonary hypertension of pre-capillary origin in WHWTs with CIPF (Roels et al., 2014).

\section{Bronchoscopy and bronchoalveolar lavage fluid analysis}

Bronchial abnormalities have been reported in a high proportion of WHWTs with CIPF, and include tracheal collapse, bronchial mucosal irregularity, an increased amount of bronchial mucus, bronchomalacia, dynamic airway collapse and bronchiectasis (Corcoran et al., 2011; Heikkilä et al., 2011; Heikkilä-Laurila and Rajamäki, 2014). These bronchial changes are not specific for CIPF, as they occur in other pulmonary diseases (Heikkilä-Laurila and Rajamäki, 2014), and some may simply be associated with old age, reflecting the population of WHWTs with CIPF (Mercier et al., 2011). Analysis of the bronchoalveolar lavage fluid (BALF) usually shows a moderate increase in the total cellular count due to 
increased numbers of macrophages, neutrophils and mast cells (Heikkilä-Laurila and Rajamäki, 2014).

\section{Diagnostic imaging}

Thoracic radiographic findings in WHWTs with CIPF are not specific and vary from a generalised broncho-interstitial pattern to only interstitial, predominantly bronchial or patchy alveolar opacities, and increased size of the heart silhouette; findings that might be observed in other pulmonary diseases (Heikkilä-Laurila and Rajamäki, 2014). Thoracic radiography can be used to assess for evidence of other pulmonary diseases, such as neoplasia (HeikkiläLaurila and Rajamäki, 2014), which has been described in a few WHWTs with CIPF.

In humans, HRCT is a standard method for providing diagnostic and prognostic information, and plays a major role in the IPF staging process (Raghu, 2011). HRCT has a high predictive value for the diagnosis of a UIP pattern. In half of human cases, HRCT is sufficient to allow a confident diagnosis of IPF. The HRCT pattern that corresponds to the pathological features of UIP includes the presence of bilateral, predominantly sub-pleural, basal reticular abnormalities, the absence of additional features considered incompatible with a diagnosis of IPF, and the presence of 'honeycombing', considered the most specific feature of the UIP pattern, with or without traction bronchiectasis (Raghu, 2011).

Several studies report HCRT findings in WHWTs with CIPF (Johnson et al., 2005; Corcoran et al., 2011; Heikkilä et al., 2011; Fitzgerald et al., 2017; Roels et al., 2017a), sometimes in comparison with findings in healthy WHWTs (Roels et al., 2017a; Thierry et al., 2017). All support the use of thoracic CT as a method for characterising IPF in WHWTs. In the first descriptions of the CT features of CIPF, common findings mainly included the presence of a ground-glass pattern and reticular abnormalities, while traction bronchiectasis and 'honeycombing' were only described in the later stages of the disease (Johnson et al., 2005; Corcoran et al., 2011; Heikkilä et al., 2011; Fitzgerald et al., 2017). In a more recent study comparing the effect of sedation and anaesthesia on thoracic HRCT findings in WHWTs (both healthy and with CIPF), and using the glossary of terms of the Fleischner Society (Roels et al., 2017a), ground-glass opacity (GGO) was identified as the most frequent lesion and was present in all image series from affected WHWTs collected both under sedation and general anaesthesia. GGO was also the only lesion observed in control age- and breed-matched dogs, but was less extensive compared with dogs with CIPF. A mosaic attenuation pattern was also frequently observed. This study concluded that despite the differences between GGO and the mosaic attenuation pattern, the use of sedation to obtain HRCT images may aid diagnosis of CIPF in WHWTs if general anaesthesia is contraindicated. Another recent study confirmed that GGO, focal reticular and mosaic attenuation patterns are the most common CT characteristics of CIPF, with the GGO pattern considered to represent mild severity, while focal reticular and mosaic attenuation patterns were associated with moderate severity (Thierry et al., 2017). Moreover, severity of pulmonary CT findings was positively correlated with severity of clinical signs and negatively associated with survival time after diagnosis (Thierry et al., 2017). These most recent HRCT studies (Roels et al., 2017a; Thierry et al., 2017) indicate that CIPF does not perfectly match IPF, but rather represents a mixed pattern with characteristics of both UIP and non-specific interstitial pneumonia (NSIP).

\section{Histopathological features}

In humans, IPF presents a histopathological image of UIP, which is characterised by areas of marked interstitial pulmonary fibrosis, 'honeycombing' and distortion of the alveolar architecture in a patchy, often sub-pleural or para-septal pattern and by characteristic fibroblastic foci at the periphery of the lesions (Katzenstein et al., 2008; Raghu, 2011). The first reports related to histopathology of CIPF in WHWTs, based on limited sample numbers, reported differences in the histopathological appearance of the pulmonary lesions in WHWTs and those characterising UIP in humans (Corcoran et al., 1999a; Norris et al., 2005; Eriksson et al., 2009; Heikkilä et al., 2011). In a later study describing the pulmonary histopathological findings in 18 WHWTs with CIPF (Syrjä et al., 2013), an underlying diffuse mature fibrosis resembling human NSIP more than UIP was seen in the lungs of all affected dogs. Additionally, the majority of dogs with CIPF showed multifocal areas of accentuated sub-pleural and peri-bronchiolar fibrosis with occasional 'honeycombing' and profound alveolar epithelial changes, reminiscent of human UIP and not commonly seen in NSIP. Interstitial fibroblastic foci, characteristic of UIP, were not seen. Therefore, CIPF in WHWTs, although presenting with histological changes typical of UIP, also shares features of both human UIP and NSIP, providing further evidence that CIPF and human IPF are not identical.

\section{Biomarkers}

Several studies have been conducted to identify biomarkers able to differentiate CIPF from other chronic lower respiratory diseases (i.e. diagnostic biomarkers), to act as a surrogate for disease severity, and/or to predict outcome (i.e. prognostic biomarkers). Although several interesting candidate biomarkers have been profiled in human IPF, large-scale multicentric studies are needed to confirm the utility of single or multiple biomarkers.

In CIPF, both screening and targeted approaches have been used to identify relevant biomarkers. Screening technologies consist of an unbiased approach, which favours exploratory identification of new biomarkers, and which can potentially lead to the identification of new disease pathways and therefore to possibilities of discovering novel anti-fibrotic agents. In this context, pulmonary gene expression by microarray analysis (Krafft et al., 2013) and analysis of the BALF proteome (Lilja-Maula et al., 2013) have been performed. Pulmonary gene expression was analysed using a specific canine microarray coupled with Ingenuity Pathway Analysis, a bioinformatics tool, in lung samples from dogs with CIPF and healthy control dogs (Krafft et al., 2013). Up-regulation of numerous genes was demonstrated, with increased expression of the chemokine-encoding genes chemokine ligand 2 (CCL2), chemokine ligand 7 (CCL7), chemokine (C-X-C motif) ligand 14 (CXCL14) and interleukin 8 (IL-8; also known as chemokine [C$\mathrm{X}-\mathrm{C}$ motif] ligand 8 [CXCL8]), and fibroblast activation protein $\alpha$ gene (FAP), confirmed by quantitative reverse transcriptase PCR (qRT-PCR). All of these genes encode proteins with potential biomarker utility. Differential gel electrophoresis and liquid chromatography-tandem mass spectrometry were used for proteomic analysis and protein identification in BALF obtained from dogs with CIPF or chronic bronchitis and healthy dogs; the study failed to identify specific biomarkers for CIPF (Lilja-Maula et al., 2013).

A targeted approach was used to select other biomarkers, based on their previously demonstrated direct or indirect pro-fibrotic effects, or based on their proven relevance as diagnostic and/or prognostic biomarkers in human IPF. Those biomarkers included procollagen type III amino terminal pro-peptide (PIIINP) (Heikkilä et al., 2013), endothelin-1 (ET-1) (Krafft et al., 2011), transforming growth factor beta-1 (TGF- $\beta 1$ ) (Krafft et al., 2014), CCL2 (Roels et al., 2015a,b), IL-8 (Roels et al., 2015a,b), 5-hydroxytryptamine- 
serotonin (5-HT) (Roels et al., 2015a), vascular endothelial growth factor (VEGF) (Roels et al., 2015a), Krebs Von den Lungen-6 (KL6) (Fastrès et al., 2018) and C-reactive-protein (CRP) (Viitanen et al., 2014). Among those biomarkers, CCL2, IL-8, ET-1 and PIIINP were elevated in the BALF of WHWTs with CIPF compared with healthy WHWTs, while only CCL2 and ET-1 were elevated in the serum of affected WHWTs compared with healthy WHWTs (Krafft et al., 2011; Heikkilä et al., 2013; Roels et al., 2015a,b). Furthermore, the blood concentrations of IL-8 (Roels et al., 2015a), TGF- $\beta 1$ (Krafft et al., 2014) and KL6 (Fastrès et al., 2018) were significantly higher in the serum of healthy WHWTs in comparison with healthy agematched dogs from breeds non-predisposed to CIPF. The presence of such high circulating concentrations of these mediators in the predisposed breed is possibly related to, or indicative of the predisposition of the WHWT breed to CIPF.

\section{Long-term outcome, prognostic indicators}

CIPF in WHWTs can have a rapid or slow disease progression (Lilja-Maula et al., 2014a) and some dogs can experience acute worsening of their respiratory function during the course of the disease (Heikkilä et al., 2013). In a study that investigated the longterm survival in WHWTs with CIPF, the median IPF-specific survival of dogs that had died was 32 months from the onset of clinical signs. According to an online questionnaire-based survey answered by 458 WHWT owners (Roels et al., 2018), the overall survival time after diagnosis was 1.4 years ( $0-8.5$ years), and cause of death was CIPF-related in $76.7 \%$ of cases.

Very few prognostic factors have been identified. Measured $\mathrm{PaO}_{2}$ and 6MWT distance were studied, but did not correlate with survival (Lilja-Maula et al., 2014a). High CCL2 blood concentration at the time of diagnosis (Roels et al., 2015a,b) and the severity of pulmonary CT findings were shown to be negatively associated with survival time after diagnosis (Thierry et al., 2017).

\section{Treatment}

At present, no effective treatments for CIPF have been described and no therapeutic trials have been performed. The current therapy aims to reduce clinical signs on an individual basis, and to alleviate possible complications that can develop during the course of the disease. Oral or inhaled corticosteroids appear to relieve cough in many dogs, particularly in the presence of concurrent bronchial changes (Corcoran et al., 1999a; Heikkilä et al., 2013). When concomitant pulmonary hypertension is present, improvement in clinical signs and a decrease in PAP after treatment with sildenafil has been reported (Kellihan et al., 2015).

In human IPF, recent anti-fibrotic therapies including pirfenidone and nintedanib have proven efficacy in slowing the course of disease (Hughes et al., 2016); however, their effect has not been documented in CIPF. Response to specific anti-fibrotic treatment has not yet been investigated in WHWTs with CIPF.

\section{Conclusions}

CIPF affects middle-aged to older WHWTs and the disease mimics human IPF, although CT and histopathological findings are not identical. Despite these dissimilarities, CIPF might be an interesting, spontaneously arising disease model for the study of human IPF. Due to the unique breed predisposition, a genetic component is suspected, but has not yet been identified. Despite numerous investigations over the past decade, the aetiopathogenesis of CIPF is not yet elucidated and no specific treatment exists, emphasising the need to identify novel therapeutic strategies in the future.

\section{Conflict of interest statement}

None of the authors of this paper has a financial or personal relationship with other people or organisations that could inappropriately influence or bias the content of the paper.

\section{Acknowledgements}

The authors wish to acknowledge Dr E. Krafft, Professor M. Rajamäki and team, and CIPF project, the Westie's breed clubs, breeders and owners are warmly thanked for their participation to the project. Part of the studies mentioned was supported by the FNRS (Fonds National de la Recherche Scientifique) and the "Fonds spéciaux pour la recherche de l'Université de Liège".

\section{References}

Allen, R.J., Porte, J., Braybrooke, R., Flores, C., Fingerlin, T.E., Oldham, J.M., GuillenGuio, B., Ma, S.F., Okamoto, T., John, A.E., et al., 2017. Genetic variants associated with susceptibility to idiopathic pulmonary fibrosis in people of European ancestry: a genome-wide association study. Lancet Respir. Med. 5, 869-880.

Antoniou, K.M., Margaritopoulos, G.A., Tomassetti, S., Bonella, F., Costabel, U., Poletti, V., 2014. Interstitial lung disease. Eur. Respir. Rev. 23, 40-54.

Balakrishnan, A., King, L.G., 2014. Updates on pulmonary function testing in small animals. Vet. Clin. North Am. Small Anim. Pract. 44, 1-18.

Corcoran, B., Cobb, M., Martin, M.W.S., French, A., Luis Fuentes, V., Boswood, A., Rhind, S., 1999a. Chronic pulmonary disease in West Highland white terriers. Vet. Rec. 144, 611-616.

Corcoran, B.M., Dukes-McEwan, J., Rhind, S., French, A., 1999b. Idiopathic pulmonary fibrosis in a Staffordshire bull terrier with hypothyroidism. J. Small Anim. Pract. 40, 185-188.

Corcoran, B.M., King, L.G., Schwarz, T., Hammond, G., Sullivan, M., 2011. Further characterisation of the clinical features of chronic pulmonary disease in West Highland white terriers. Vet. Rec. 168, 355.

Daccord, C., Maher, T.M., 2016. Recent advances in understanding idiopathic pulmonary fibrosis. F1000Research 5, 1046.

Demir, R., Küçükoglu, M.S., 2015. Six-minute walk test in pulmonary arterial hypertension. Anatol. J. Cardiol. 15, 249-254.

Eriksson, M., von Euler, H., Ekman, E., Nordling, K., Häggström, J., Johansson, J., 2009. Surfactant protein C in canine pulmonary fibrosis. J. Vet. Intern. Med. 23, 11701174.

Fastrès, A., Roels, E., Bolen, G., Tutunaru, A., Antoine, N., Farnir, F., Clercx, C., 2018. Investigation of serum Krebs Von den Lungen 6 (KL-6) concentration as a predisposing factor and in the diagnosis of canine idiopathic pulmonary fibrosis in the West Highland white terrier. Proceedings of the 28th ECVIM-CA Congress, Rotterdam, 6th-8th September.

Fingerlin, T.E., Murphy, E., Zhang, W., Peljto, A.L., Brown, K.K., Steele, M.P., Loyd, J.E., Cosgrove, G.P., Lynch, D., Groshong, S., et al., 2013. Genome-wide association study identifies multiple susceptibility loci for pulmonary fibrosis. Nat. Genet. 45, 613-620.

Fitzgerald, E., Priestnall, S.L., Lamb, C.R., 2017. Imaging diagnosis-computed tomography of traction bronchiectasis secondary to pulmonary fibrosis in a patterdale terrier. Vet. Radiol. Ultrasound 58, E42-E44.

Heikkilä-Laurila, H.P., Rajamäki, M.M., 2014. Idiopathic pulmonary fibrosis in West Highland white terriers. Vet. Clin. North Am. Small Anim. Pract. 44, 129-142.

Heikkilä, H.P., Krafft, E., Jespers, P., McEntee, K., Rajamäki, M.M., Clercx, C., 2013. Procollagen type III amino terminal propeptide concentrations in dogs with idiopathic pulmonary fibrosis compared with chronic bronchitis and eosinophilic bronchopneumopathy. Vet. J. 196, 52-56.

Heikkilä, H.P., Lappalainen, A.K., Day, M.J., Clercx, C., Rajamäki, M.M., 2011. Clinical, bronchoscopic, histopathologic, diagnostic imaging, and arterial oxygenation findings in West Highland white terriers with idiopathic pulmonary fibrosis. J. Vet. Intern. Med. 25, 433-439.

Hughes, G., Toellner, H., Morris, H., Leonard, C., Chaudhuri, N., 2016. Real world experiences: pirfenidone and nintedanib are effective and well tolerated treatments for idiopathic pulmonary fibrosis. J. Clin. Med. 5, 78.

Johnson, V.S., Corcoran, B.M., Wotton, P.R., Schwarz, T., Sullivan, M., 2005. Thoracic high-resolution computed tomographic findings in dogs with canine idiopathic pulmonary fibrosis. J. Small Anim. Pract. 46, 381-388.

Katzenstein, A.L.A., Mukhopadhyay, S., Myers, J.L., 2008. Diagnosis of usual interstitial pneumonia and distinction from other fibrosing interstitial lung diseases. Hum. Pathol. 39, 1562-1581.

Kellihan, H.B., Waller, K.R., Pinkos, A., Steinberg, H., Bates, M.L., 2015. Acute resolution of pulmonary alveolar infiltrates in 10 dogs with pulmonary hypertension treated with sildenafil citrate: 2005-2014. J. Vet. Cardiol. 17, 182191.

Kouranos, V., Jacob, J., Nicholson, A., Renzoni, E., 2017. Fibrotic hypersensitivity pneumonitis: key issues in diagnosis and management. J. Clin. Med. 6, 62.

Krafft, E., Heikkilä, H.P., Jespers, P., Peeters, D., Day, M.J., Rajamäki, M.M., Mc Entee, K., Clercx, C., 2011. Serum and bronchoalveolar lavage fluid endothelin-1 
concentrations as diagnostic biomarkers of canine idiopathic pulmonary fibrosis. J. Vet. Intern. Med. 25, 990-996.

Krafft, E., Laurila, H.P., Peters, I.R., Bureau, F., Peeters, D., Day, M.J., Rajamäki, M.M., Clercx, C., 2013. Analysis of gene expression in canine idiopathic pulmonary fibrosis. Vet. J. 198, 479-486.

Krafft, E., Lybaert, P., Roels, E., Laurila, H.P., Rajamäki, M.M., Farnir, F., Myllärniemi, M., Day, M.J., Mc Entee, K., Clercx, C., 2014. Transforming growth factor beta 1 activation, storage, and signaling pathways in idiopathic pulmonary fibrosis in dogs. J. Vet. Intern. Med. 28, 1666-1675.

Lilja-Maula, L., Syrjä, P., Laurila, H.P., Sutinen, E., Palviainen, M., Ritvos, O., Koli, K., Rajamäki, M.M., Myllärniemi, M., 2015. Upregulation of alveolar levels of activin $\mathrm{B}$, but not activin A, in lungs of West Highland white terriers with idiopathic pulmonary fibrosis and diffuse alveolar damage. J. Comp. Pathol. 152, 192-200.

Lilja-Maula, L.I.O., Laurila, H.P., Syrjä, P., Lappalainen, A.K., Krafft, E., Clercx, C., Rajamäki, M.M., 2014a. Long-term outcome and use of 6-minute walk test in West Highland white terriers with idiopathic pulmonary fibrosis. J. Vet. Intern. Med. 28, 379-385.

Lilja-Maula, L., Syrjä, P., Laurila, H.P., Sutinen, E., Rönty, M., Koli, K., Rajamäki, M.M., Myllärniemi, M., 2014b. Comparative study of transforming growth factor- $\beta$ signalling and regulatory molecules in human and canine idiopathic pulmonary fibrosis. J. Comp. Pathol. 150, 399-407.

Lilja-Maula, L.I., Palviainen, M.J., Heikkila, H.P., Raekallio, M.R., Rajamäki, M.M., 2013. Proteomic analysis of bronchoalveolar lavage fluid samples obtained from West Highland white terriers with idiopathic pulmonary fibrosis, dogs with chronic bronchitis, and healthy dogs. Am. J. Vet. Res. 74, 148-154.

Lobetti, R.G., Milner, R., Lane, E., 2001. Chronic idiopathic pulmonary fibrosis in five dogs. J. Am. Anim. Hosp. Assoc. 37, 119-127.

Lynch, D.A., Sverzellati, N., Travis, W.D., Brown, K.K., Colby, T.V., Galvin, J.R., Goldin, J. G., Hansell, D.M., Inoue, Y., Johkoh, T., et al., 2018. Diagnostic criteria for idiopathic pulmonary fibrosis: a Fleischner Society White Paper. Lancet Respir. Med. 6, 138-153.

Maatta, M., Laurila, H., Holopainen, S., Lilja-Maula, L., Melamies, M., Viitanen, S., Johnson, L., Koho, N., Neuvonen, M., Niemi, M., et al., 2018. Reflux aspiration in lungs of dogs with respiratory disease and in healthy West Highland white terriers. J. Vet. Intern. Med. 12 (October), e15321. doi:http://dx.doi.org/10.1111/ jvim.15321 [Epub ahead of print].

Mercier, E., Bolognin, M., Hoffmann, A.C., Tual, C., Day, M.J., Clercx, C., 2011. Influence of age on bronchoscopic findings in healthy beagle dogs. Vet. J. 187, 225-228.

Minnis, P., Henry, K., Keane, M.P., 2016. Reflux in idiopathic pulmonary fibrosis. Q. J. Med. 109, 7-10.

Molyneaux, P.L., Maher, T.M., 2013. The role of infection in the pathogenesis of idiopathic pulmonary fibrosis. Eur. Respir. Rev. 22, 376-381.

Neurohr, C., Behr, J., 2015. Changes in the current classification of IIP: a critical review. Respirology 20, 699-704.

Norris, A.J., Naydan, D.K., Wilson, D.W., 2005. Interstitial lung disease in West Highland white terriers. Vet. Pathol. 42, 35-41.

Norris, C.R., Griffey, S.M., Walsh, P., 2002. Use of keyhole lung biopsy for diagnosis of interstitial lung diseases in dogs and cats: 13 cases (1998-2001). J. Am. Vet. Med. Assoc. 221, 1453-1459.

Olson, A.L., Swigris, J.J., 2012. Idiopathic pulmonary fibrosis: diagnosis and epidemiology. Clin. Chest Med. 33, 41-50.

Raghu, G., 2011. Idiopathic pulmonary fibrosis: guidelines for diagnosis and clinical management have advanced from consensus-based in 2000 to evidence-based in 2011. Eur. Respir. J. 37, 743-746.

Reinero, C.R., Cohn, L.A., 2007. Interstitial lung diseases. Vet. Clin. North Am. Small Anim. Pract. 37, 937-947.

Roels, E., Couvreur, T., Farnir, F., Clercx, C., Verschakelen, J., Bolen, G., 2017a. Comparison between sedation and general anesthesia for high-resolution computed tomographic characterization of canine idiopathic pulmonary fibrosis in West Highland white terriers. Vet. Radiol. Ultrasound 58, 284-294.

Roels, E., Barrera, C., Millon, L., Rajamäki, M., Talbot, J., Clercx, C., Barrs, V., 2017b. Investigation of a fungal aetiology in canine idiopathic pulmonary fibrosis. Proceedings of the 27th ECVIM-CA Congress, St Julian's, Malta, 14th-16th September.

Roels, E., Dourcy, M., Holopainen, S., Rajamäki, M.M., Gillet, L., Ehlers, B., Clercx, C., 2016. No evidence of herpesvirus infection in West Highland white terriers with canine idiopathic pulmonary fibrosis. Vet. Pathol. 53, 1210-1212.

Roels, E., Fastrès, A., McGeown, P., Gommeren, K., Saegerman, C., Clercx, C., 2018. Questionnaire-based survey of owner-reported environment and care of West Highland white terrier with or without idiopathic pulmonary fibrosis. Proceedings of the 28th ECVIM-CA Congress, Rotterdam, 6th-8th September.

Roels, E., Krafft, E., Antoine, N., Farnir, F., Laurila, H.P., Holopainen, S., Rajamäki, M.M. Clercx, C., 2015a. Evaluation of chemokines CXCL8 and CCL2, serotonin, and vascular endothelial growth factor serum concentrations in healthy dogs from seven breeds with variable predisposition for canine idiopathic pulmonary fibrosis. Res. Vet. Sci. 101, 57-62.

Roels, E., Krafft, E., Farnir, F., Holopainen, S., Laurila, H.P., Rajamäki, M.M., Day, M.J., Antoine, N., Pirottin, D., Clercx, C., 2015b. Assessment of CCL2 and CXCL8 chemokines in serum, bronchoalveolar lavage fluid and lung tissue samples from dogs affected with canine idiopathic pulmonary fibrosis. Vet. J. 206, 75-82.

Roels, E., Merveille, A., Couvreur, T., Bolen, G., Krafft, E., Clercx, C., Mc Entee, K., 2014. Correlation between thoracic CT-scan angiography findings and echocardiographic right pulmonary vein to pulmonary artery ratio in West Highland white terriers with idiopathic pulmonary fibrosis. Proceedings of the 24th ECVIM-CA Congress, Mainz, Germany, 4th-6th September.

Schober, K.E., Baade, H., 2006. Doppler echocardiographic prediction of pulmonary hypertension in West Highland white terriers with chronic pulmonary disease. J. Vet. Intern. Med. 20, 912-920.

Serres, F., Chetboul, V., Gouni, V., Tissier, R., Sampedrano, C.C., Pouchelon, J.-L., 2007. Diagnostic value of echo-Doppler and tissue Doppler imaging in dogs with pulmonary arterial hypertension. J. Vet. Intern. Med. 21, 1280-1289.

Sgalla, G., Biffi, A., Richeldi, L., 2016. Idiopathic pulmonary fibrosis: diagnosis, epidemiology and natural history. Respirology 21, 427-437.

Spagnolo, P., Rossi, G., Cavazza, A., Bonifazi, M., Paladini, I., Bonella, F., Sverzellati, N., Costabel, U., 2015. Hypersensitivity pneumonitis: a comprehensive review. J. Investig. Allergol. Clin. Immunol. 25, 237-250.

Syrjä, P., Heikkilä, H.P., Lilja-Maula, L., Krafft, E., Clercx, C., Day, M.J., Rönty, M. Myllärniemi, M., Rajamäki, M.M., 2013. The histopathology of idiopathic pulmonary fibrosis in West Highland white terriers shares features of both nonspecific interstitial pneumonia and usual interstitial pneumonia in man. J. Comp. Pathol. 149, 303-313.

Thierry, F., Handel, I., Hammond, G., King, L.G., Corcoran, B.M., Schwarz, T., 2017. Further characterization of computed tomographic and clinical features for staging and prognosis of idiopathic pulmonary fibrosis in West Highland white terriers. Vet. Radiol. Ultrasound 58, 381-388.

Viitanen, S.J., Laurila, H.P., Lilja-Maula, L.I., Melamies, M.A., Rantala, M., Rajamäki, M. M., 2014. Serum C-reactive protein as a diagnostic biomarker in dogs with bacterial respiratory diseases. J. Vet. Intern. Med. 28, 84-91.

Visser, L.C., Im, M.K., Johnson, L.R., Stern, J.A., 2016. Diagnostic value of right pulmonary artery distensibility index in dogs with pulmonary hypertension: comparison with Doppler echocardiographic estimates of pulmonary arterial pressure. J. Vet. Intern. Med. 30, 543-552.

Webb, J.A., Armstrong, J., 2002. Chronic idiopathic pulmonary fibrosis in a West Highland white terrier. Can. Vet. J. 43, 703-705. 